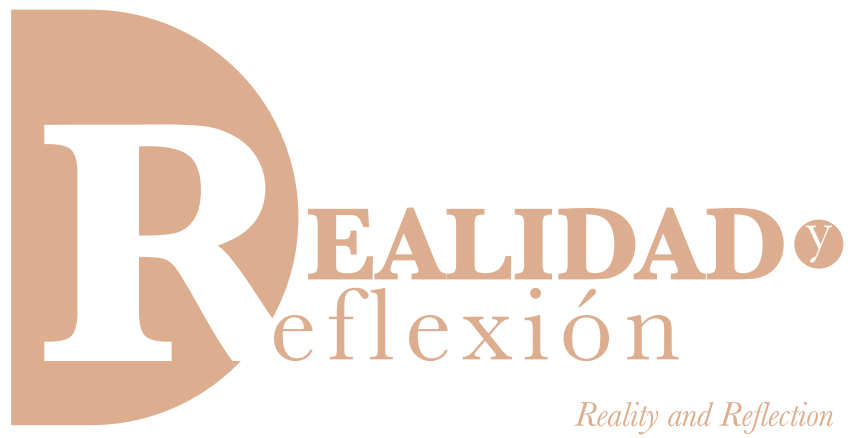

ISSN 1992-6510

e-ISSN 2520-9299

Año 17, N 46, San Salvador, El Salvador, Centroamérica. Revista Semestral Julio-Diciembre 2017

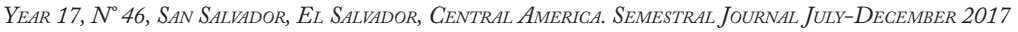

\title{
Matrícula y titulación de educación superior en E1 Salvador, con perspectiva de género
}

\author{
Enrollment and qualification of higher education in \\ El Salvador, with gender perspective
}

\author{
Ana Milena Marquina de Reyes ${ }^{1}$ \\ Doctora en Medicina, Maestra en Educación Superior \\ Maestra en Salud Pública, Directora de Educación Médica, Facultad de \\ Medicina, Universidad Evangélica de E1 Salvador \\ milena.reyes@uees.edu.sv \\ Mirna Maribel García de González \\ Doctora en Medicina, Maestra en Educación Superior, \\ Directora de Evaluación y Acreditación, Universidad \\ Evangélica de El Salvador \\ mirna.garcia@uees.edu.sv \\ Recibido: 24 de julio de 2017 \\ Aprobado: 13 de diciembre de 2017 \\ pág. $54-66$ \\ DOI: http://dx.doi.org/10.5377/ryr.v0i46.5507
}

\section{RESUMEN}

El articulo analiza la matrícula y la titulación de la educación superior en El Salvador desde la óptica de perspectiva de género, examinando la tendencia en el quinquenio 2010 - 2014 visualizando que la matrícula a este nivel presenta una equidad por otra parte la titulación de pregrado presenta una feminización, además se hace un recorrido sobre los datos que presentan las instituciones de educación superior al Ministerio de educación sobre la matrícula y titulación de posgrado, dichos datos muestran una equidad del género femenino. Por último se plantea el analices sobre la planta docente universitario por sexo y su titulación de posgrado, donde los datos se inclinan a favor del género masculino.

Palabras clave: Educación superior, feminización, docente, titulación, matrícula, pregrado, postgrado.

\section{ABSTRACT}

The article analyzes the enrollment and qualification of higher education in El Salvador from the point of view about gender, testing the trend the quinquennium 2010-2014 visualizing the enrollment at this level shows an equity. On the other hand the enrollment certification degree presents a feminization besides a tour of the data presented by the institutions of higher education to the Ministry of education on the enrollment and graduate degree, such data show an equiaty of the female gender. Finally, it is considered the analusis of the universal educational establishment by sex and its potgraduate degree where the data are inclined in favor of the male gender.

Keywords: Higher education, feminization, professor, qualification, enrollment, undergraduate, postgraduate. 


\section{Introducción}

Tradicionalmente, la educación superior no fue un espacio considerado propiamente "femenino", constituyendo uno de los ámbitos privilegiados de reproducción de las desigualdades de género en el fortalecimiento de la división sexual del trabajo, mientras el género femenino eran invisibilizadas en la esfera privada y asignadas a las tareas propias de la reproducción y cuidados de la familia y el hogar.

En este escenario el género femenino ha sido, históricamente, menos favorecidas en su inserción en el sistema educativo y, en consecuencia, en las oportunidades que este ofrece. De ahí que "feminización" se tomará tanto ya sea la matrícula o la titulación sea mayor frente a la matrícula o titulación del género masculino.

Además de la feminización de la matrícula de la educación superior en América Latina y el Caribe, se ha observado, en todos los países una tendencia consistente en la superación de algunas de las barreras entre tipos de estudios considerados "femeninos" o "masculinos". Esto expresa un fenómeno de transición cultural de gran relevancia para la igualdad de oportunidades de género tanto a nivel internacional como del país.

Aun así, continúan existiendo carreras o áreas de estudio consideradas propiamente "femeninas" en tanto el desempeño de las mismas permanece asociado a la extensión de los papeles tradicionales de la mujer en el espacio privado, tales como el cuidado y atención de personas dependientes y tareas relacionadas con la alimentación, educación básica, salud primaria, relaciones públicas, entre otros. Enfermería, nutrición, educación básica y media, ciencias sociales, entre otras opciones educativas, son relacionadas a roles considerados culturalmente como femeninos y continúan siendo espacios de concentración de la matrícula femenina en la educación superior.

Las dificultades de superación de estas barreras culturales en la inserción de género masculino y femenino en la educación superior no son fácilmente superables. Por el contrario, la segregación de género en la educación superior, en algunas disciplinas, reproduce y mantiene la desigualdad en el mercado laboral.

Cabe recalcar la importancia de indagar sobre esta problemática ya que a nivel de grupo poblacional, el género femenino es cuantitativamente superior que el masculino, principalmente en las edades donde pueden desarrollarse profesionalmente $\mathrm{y}$ obtener un título universitario.

Agregando a lo anterior es interesantes realizarnos la siguiente interrogante ¿Es la matrícula y titulación en educación superior a predominio femenino, así como hay áreas del conocimiento específicas para el género femenino?

\section{Desarrollo}

El análisis de la matrícula y titulación de El Salvador se desarrolló a través de la técnica de la observación de una recopilación de información estadística del Ministerio de Educación sobre los tópicos antes mencionados en las instituciones de educación superior (IES), así como también por sexo y área de conocimiento. 


\section{Área de Pregrado: Matrícula}

Al hablar de Pregrado se entenderá al estudiantado que está oficialmente registrado en un programa de estudio en una institución de educación superior. El Gráfico n. 1 muestra la matrícula para el quinquenio $(2010$ - 2014) con perspectiva de género.

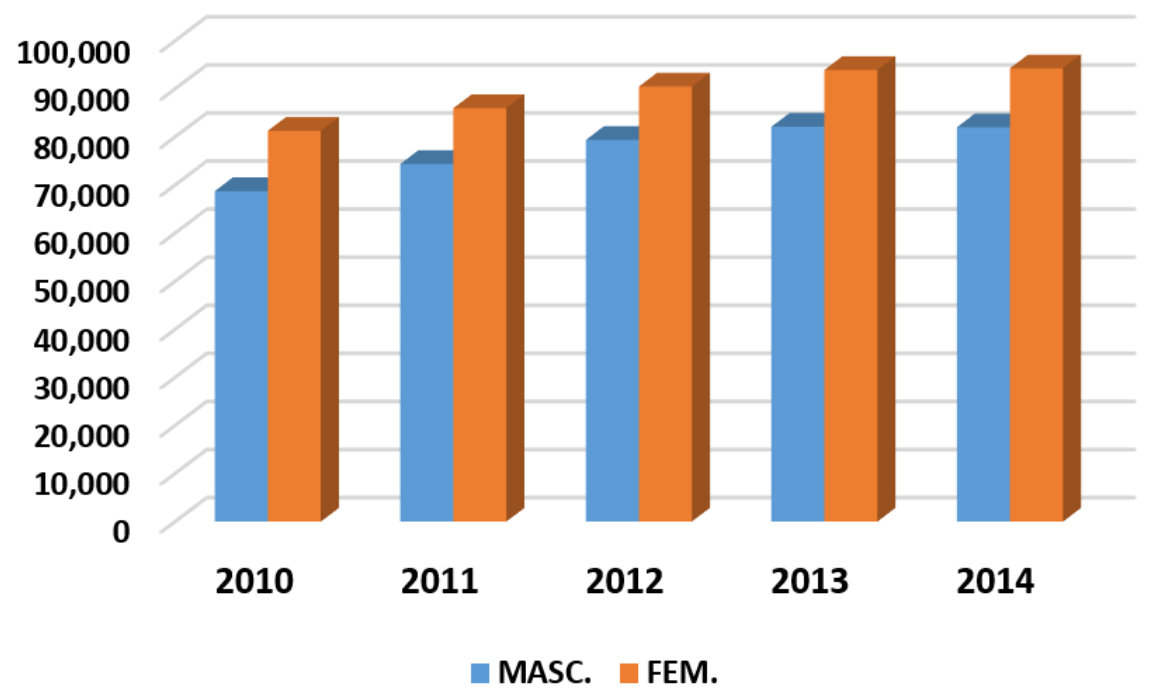

Gráfico n. ${ }^{\circ}$ 1. Matrícula por género en educación superior (2010-2014). Fuente: Elaboración propia con datos estadísticos del Ministerio de Educación 2010-2014.

De acuerdo a la información entregada por las (IES) a la Dirección Nacional de Educación Superior (DNSE) del Ministerio de Educación, la matrícula total de educación superior en los años 2010 a 2014 alcanzó un total de 150,012 a 176,293 de estudiantado respectivamente. De ese total, el 54\% corresponde a matrícula del género femenino en el año 2010 y 53\% en el año 2014.

Según la Organización de las Naciones Unidas para la Educación, la Ciencia y la Cultura (UNESCO) 2012, la tasa bruta de matrícula (TBM) en la enseñanza superior en los 158 países de los que hay datos. Es inferior al 20\% en un $43 \%$ de los países, en una cuarta parte de ellos se sitúa entre el 20\% y el $50 \%$ y otro cuarto (un 26\%) registra tasas del
$50 \%$ al 80\%. En sólo nueve países la tasa es superior al 80\%. Partiendo de estos datos de 2010 a 2014, El Salvador paso de una TBM de un $24.99 \%$ al $25.75 \%$, situándonos en la cuarta parte de los países con una TBM entre el $20 \%$ y el $50 \%$.

En el Gráfico n. 2 se observa que, desde el año 2010, la participación femenina tuvo su punto máximo de 54.17\%, teniendo una baja en el año 2012, contando con un leve aumento para el año 2014 con 53.47\%. En este sentido, se rompe la tendencia de aumento sostenido de la participación de las mujeres en la matrícula de educación superior, evidenciándose un estancamiento en términos porcentuales de participación segmentando por género. 


\section{Matrícula Total de Estudiantes en Instituciones de Educación Superior por género y participación de mujeres (\%), período 2010 - 2014}

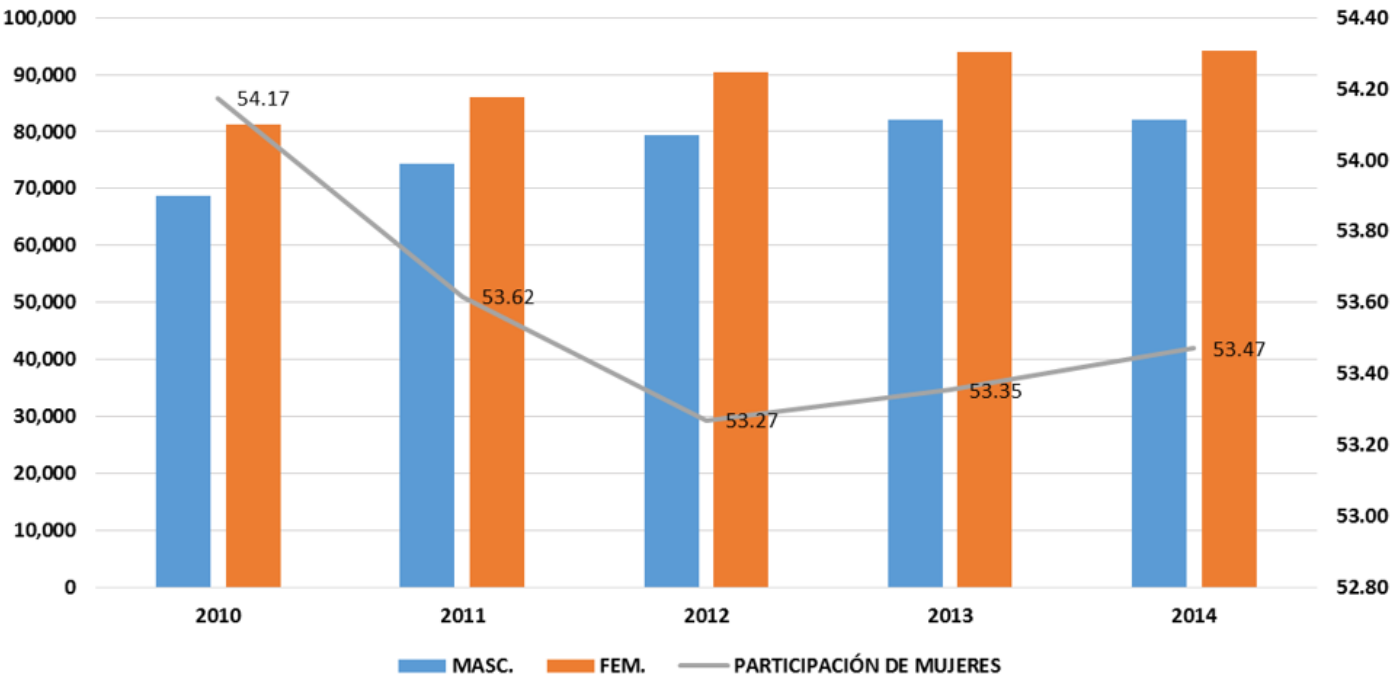

Gráfico $n .^{\circ}$ 2. Matrícula total de educación superior por género y participación de mujeres. Fuente: Elaboración propia con datos estadísticos del Ministerio de Educación 2010-2014.

Respecto a la brecha de matrícula total entre el género masculino y femenino para el 2014, esta alcanza un valor de 6 puntos porcentuales, en donde el género masculino, desde el año 2010, ha perdido la predominancia en la participación en matrícula de educación superior.

Tabla n. ${ }^{\circ} 1$

Matrícula total según área de conocimiento

\begin{tabular}{|c|c|c|c|c|c|c|c|c|c|c|c|c|c|c|c|}
\hline \multirow{2}{*}{ ÁREA DE FORMACIÓN } & \multicolumn{3}{|c|}{ AÑO 2010} & \multicolumn{3}{|c|}{ AÑO 2011} & \multicolumn{3}{|c|}{ AÑO 2012} & \multicolumn{3}{|c|}{ AÑO2013 } & \multicolumn{3}{|c|}{ AÑO 2014} \\
\hline & MASC. & FEM. & TOTAL & MASC. & FEM. & TOTAL & MASC. & FEM. & TOTAL & MASC. & FEM. & TOTAL & MASC. & FEM. & TOTAL \\
\hline Arte y Arquitectura & 2,955 & 2,519 & 5,474 & 3,486 & 2,924 & 6,410 & 3,737 & 3,134 & 6,871 & 3,931 & 3,418 & 7,349 & 3,979 & 3,759 & 7,738 \\
\hline Economía, Administración y Comercio & 15,883 & 22,628 & 38,511 & 17,085 & 24,110 & 41,195 & 18,202 & 25,030 & 43,232 & 18,672 & 25,664 & 44,336 & 18,921 & 25,824 & 44,745 \\
\hline Salud & 6,812 & 18,526 & 25,338 & 7,776 & 20,218 & 27,994 & 8,602 & 21,849 & 30,451 & 9,375 & 23,682 & 33,057 & 9,623 & 24,276 & 33,899 \\
\hline Ciencias & 1,185 & 1,525 & 2,710 & 1,289 & 1,535 & 2,824 & 1,379 & 1,690 & 3,069 & 1,450 & 1,759 & 3,209 & 1,481 & 1,742 & 3,223 \\
\hline Agropecuaria y Medio Ambiente & 1,536 & 777 & 2,313 & 1,718 & 825 & 2,543 & 1,925 & 896 & 2,821 & 2,103 & 999 & 3,102 & 2,239 & 1,126 & 3,365 \\
\hline Derecho & 6,832 & 8,815 & 15,647 & 6,873 & 8,759 & 15,632 & 6,955 & 8,713 & 15,668 & 7,115 & 8,909 & 16,024 & 6,888 & 8,830 & 15,718 \\
\hline Humanidades & 2,694 & 3,649 & 6,343 & 3,341 & 4,535 & 7,876 & 3,835 & 5,127 & 8,962 & 4,218 & 5,771 & 9,989 & 4,382 & 6,149 & 10,531 \\
\hline Tecnología & 24,020 & 8,107 & 32,127 & 25,222 & 8,067 & 33,289 & 26,447 & 8,032 & 34,479 & 27,003 & 7,837 & 34,840 & 26,406 & 7,493 & 33,899 \\
\hline Educación & 4,199 & 10,420 & 14,619 & 4,777 & 10,458 & 15,235 & 5,306 & 11,041 & 16,347 & 5,031 & 10,672 & 15,703 & 4,752 & 9,589 & 14,341 \\
\hline Ciencias Sociales & 2,628 & 4,302 & 6,930 & 2,819 & 4,557 & 7,376 & 2,988 & 4,972 & 7,960 & 3,227 & 5,227 & 8,454 & 3,355 & 5,479 & 8,834 \\
\hline TOTAL & 68,744 & 81,268 & 150,012 & 74,386 & 85,988 & 160,374 & 79,376 & 90,484 & 169,860 & 82,125 & 93,938 & \begin{tabular}{|l|}
176,063 \\
\end{tabular} & 82,026 & 94,267 & 176,293 \\
\hline Porcentaje & 45.83 & 54.17 & 100 & \begin{tabular}{|l|}
46.38 \\
\end{tabular} & 53.62 & 100 & 46.73 & 53.27 & 100 & 46.65 & 53.35 & 100 & 46.53 & 53.47 & 100 \\
\hline
\end{tabular}

Fuente: Elaboración propia con datos estadísticos del Ministerio de Educación 2010-2014. 
Según datos mostrados, para el año 2014, el género femenino muestra una participación mayoritaria en casi todas las áreas del conocimiento, con excepción de las áreas: agropecuaria y medio ambiente (33.46\%), arte y arquitectura (48.58\%), y tecnología (22.10\%).
En el caso de tecnología, ésta es ampliamente dominada por el género masculino, con el 77.9\% de la matrícula. Por otro lado, salud (71.61\%), educación $(66.86 \%)$ y ciencias sociales $(62 \%)$ son las áreas de conocimiento con más alta participación femenina.

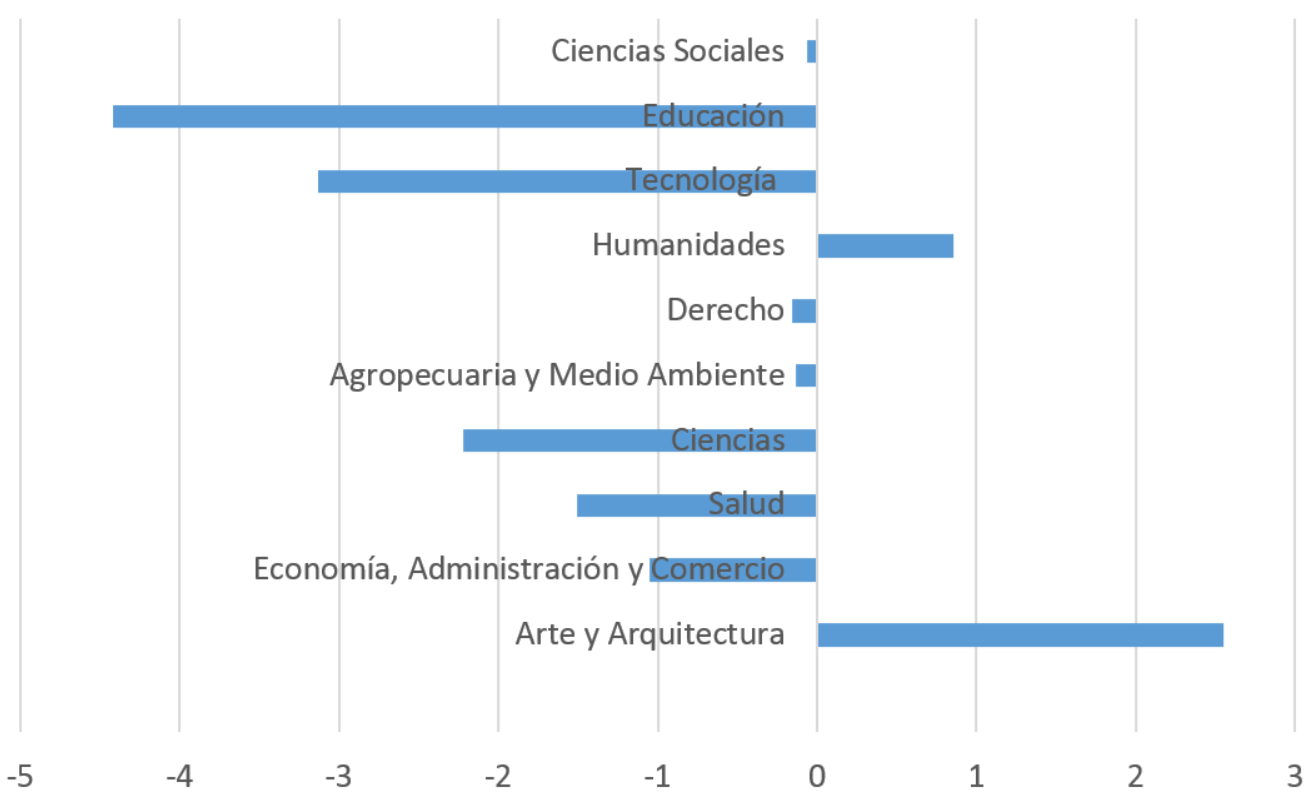

Gráfico n. ${ }^{\circ}$ 3. Puntos porcentuales en matrícula en mujeres 2010-2014. Fuente: Elaboración propia con datos estadísticos del Ministerio de Educación 2010-2014.

En el Gráfico n. 3 se analizan las áreas del conocimiento en las cuales se presenta un crecimiento en el porcentaje de participación femenina, en términos de puntos porcentuales: arte y cultura 2 puntos, humanidades ha aumentado cerca de 1 punto porcentual en el quinquenio en estudio, el resto de las áreas de conocimiento han bajado los puntos porcentuales desde el área de ciencias sociales con menos 0.06 puntos hasta en educación con menos 4 puntos porcentuales. En este sentido, podría esperarse que género femenino siga aumentando sustancialmente su participación en estas áreas del conocimiento.

Según Busto (2004), el ingreso mayor de mujeres a la educación superior, en comparación con los hombres, no implica que ha desaparecido la división de carreras femeninas y masculinas. Si bien cada vez ingresan más mujeres a las diferentes áreas del conocimiento, todavía los porcentajes mayores se observan en letras, humanidades y ciencias sociales. 
Para la UNESCO (2012), en ciertos casos los hombres pueden tener más probabilidad que las mujeres de pasar directamente de la enseñanza secundaria al mundo laboral o a la educación no formal, o bien de proseguir los estudios en el extranjero. Pero la tendencia de fondo que a largo plazo lleva a una inflexión y un creciente predominio de la matrícula femenina responden a un cambio de mentalidad en el terreno social y familiar acerca de la educación de la mujer.
El indicador apropiado para analizar la disparidad entre género es el Índice de Paridad de Género (IPG). En su versión más simple se calcula como el cociente entre el número de mujeres y el número de hombres. Este indicador mide el avance hacia la paridad entre los géneros en términos de la participación en la educación. Es un buen indicador para observar los avances de la participación femenina en los estudios universitarios.

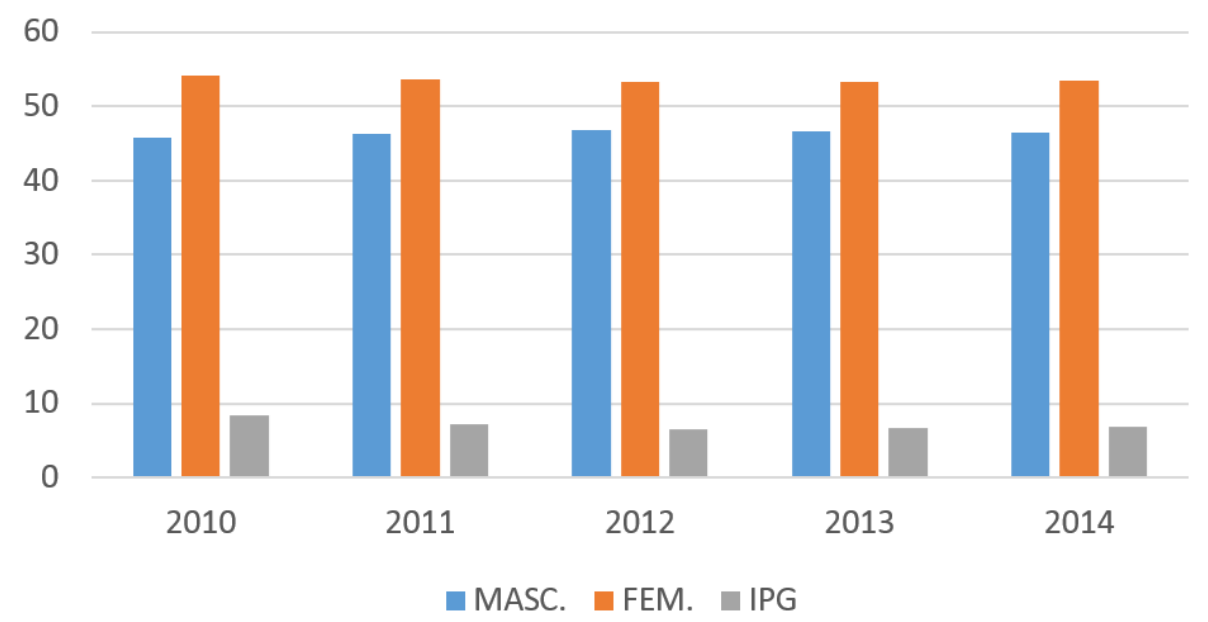

Gráfico n. ${ }^{\circ}$ 4. Matrícula por sexo e índice de paridad de género. Fuente: Elaboración propia con datos estadísticos del Ministerio de Educación 2010-2014.

En el Gráfico n. ${ }^{\circ}$ 4, El IPG obtenido de los números totales de matrícula por género sin desagregar, nos muestran que la disparidad de género beneficia a las mujeres en todos los años del quinquenio en estudio. Dicho índice fue más pronunciado para el año 2010 y ha tenido un leve descenso en los años siguientes.

A lo largo de los años el porcentaje de mujeres matriculadas y tituladas del subsistema de educación superior, es mayor al de los hombres.
Este fenómeno se denomina "feminización" de la educación superior.

Este progreso se debe a estrategias que han centrado la atención en el problema de las desigualdades de género (Busto, 2004). Papadópulos y Radakovich (2006) opinan que la educación superior en el mundo ha jugado un papel protagónico en la búsqueda de consolidar estructuras igualitarias de oportunidades entre hombres y mujeres. 
Papadópulos y Radakovich (2006), clasifica la feminización en incipiente, cuando menos del $47 \%$ de la matrícula es femenina, en equidad, cuando la matrícula femenina oscila entre el 47 $\%$ y $54 \%$ y la propiamente dicha cuando esta es mayor al 54 \%., contando en El Salvador la participación femenina en la matricula en 2010 de 54.17\%, y para el año 2014 con 53.47\%.

La feminización incipiente, donde aún no se alcanza la equidad en la matriculación. Esto es un indicador de las dificultades de cambiar patrones históricos de distribución de roles entre hombres y mujeres. Un segundo grupo de países, en el cual el nivel de matriculación de uno u otro sexo es similar o levemente superior al 50\% (en más o menos 3\%) en relación al otro, muestra equidad de género. Por lo anterior expuesto como en el 2014 El Salvador tiene una feminización que logra la equidad en la matriculación sin llegar aun al Feminización propiamente de la matrícula.

\section{Titulación Pregrado}

Al analizar la participación por género en las titulaciones en las distintas áreas del conocimiento, se observa que para el año 2014 las mujeres muestran una participación mayoritaria en casi todas las áreas, con la excepción de agropecuaria y medio ambiente y tecnología, en donde la participación femenina en la primera es de $27.57 \%$ y en la segunda de $24.84 \%$.

Las áreas con mayor titulación de género femenino son: salud (77.41\%), educación (65.29\%) y ciencias sociales (67.44\%). Esta situación va en la misma línea con lo que ocurre en matrícula, donde las tendencias en áreas de conocimiento son muy similares al momento de aplicar un análisis con perspectiva de género.

Tabla n. ${ }^{\circ} 2$

Titulación por nivel universitario según área de conocimiento

\begin{tabular}{|c|c|c|c|c|c|c|c|c|c|c|c|c|c|c|c|}
\hline \multirow{2}{*}{ ÁREA } & \multicolumn{3}{|c|}{ AÑO 2010} & \multicolumn{3}{|c|}{ AÑO 2011} & \multicolumn{3}{|c|}{ AÑO 2012} & \multicolumn{3}{|c|}{ AÑO 2013} & \multicolumn{3}{|c|}{ AÑO 2014} \\
\hline & MASC & FEM & TOTAL & MASC & FEM & TOTAL & MASC & FEM & TOTAL & MASC & FEM & TOTAL & MASC & FEM & TOTAL \\
\hline Arte y Arquitectura & 219 & 280 & 499 & 260 & 271 & 531 & 312 & 325 & 637 & 307 & 305 & 612 & 312 & 315 & 627 \\
\hline Economía, Administración y Comercio & 1,467 & 2,265 & 3,732 & 1,659 & 2,812 & 4,471 & 1,855 & 2,989 & 4,844 & 2,169 & 3,462 & 5,631 & 2,111 & 3,384 & 5,495 \\
\hline Salud & 628 & 2,602 & 3230 & 761 & 2,771 & 3,532 & 868 & 3,012 & 3,880 & 887 & 3,139 & 4,026 & 966 & 3,312 & 4,278 \\
\hline Ciencias & 67 & 111 & 178 & 72 & 93 & 165 & 90 & 123 & 213 & 98 & 141 & 239 & 93 & 125 & 218 \\
\hline Agropecuaria y Medio Amb & 129 & 57 & 186 & 200 & 73 & 273 & 224 & 82 & 306 & 266 & 87 & 353 & 239 & 91 & 330 \\
\hline Derecho & 688 & 1,007 & 1695 & 725 & 1,019 & 1,744 & 746 & 1,109 & 1,855 & 796 & 1,090 & 1,886 & 762 & 1,039 & 1,801 \\
\hline Humanidades & 139 & 184 & 323 & 178 & 239 & 417 & 209 & 268 & 477 & 191 & 296 & 487 & 226 & 309 & 535 \\
\hline Tecnología & 2,916 & 1,156 & 4,072 & 3,230 & 1,293 & 4,523 & 3,241 & 1,212 & 4,453 & 3,677 & 1,334 & $\begin{array}{l}4,011 \\
\end{array}$ & 3,687 & 1,217 & 4,904 \\
\hline Educación & 610 & 1,945 & 2555 & 1,084 & 2,818 & 3,902 & 1,222 & 2,910 & 4,132 & 1,560 & 2,852 & 4,412 & 1,441 & 2,711 & 4,152 \\
\hline Ciencias Sociales & 188 & 431 & 619 & 202 & 524 & 726 & 284 & 585 & 869 & 304 & 658 & \begin{tabular}{|l|l|} 
& 962 \\
\end{tabular} & 278 & 576 & 854 \\
\hline TOTAL & 7,051 & 10,038 & 17,089 & 8,371 & 11,913 & 20,284 & 9,051 & 12,615 & 21,666 & 10,255 & 13,364 & $\begin{array}{r}23,619 \\
\end{array}$ & 10,115 & 13,079 & 23,194 \\
\hline Porcentaje & 41.26 & 58.74 & 100 & 41.26 & 58.74 & 100 & 41.27 & 58.73 & 100 & 41.78 & 58.22 & 100 & 43.61 & 56.39 & 100 \\
\hline
\end{tabular}

Fuente: Elaboración propia con datos estadísticos del Ministerio de Educación 2010-2014. 
En la Tabla n. ${ }^{\circ} 2$ es posible revisar longitudinalmente la participación femenina en la titulación por área de conocimiento, donde es posible observar que en un número importante de áreas el género femenino presenta un aumento sostenido en todas las áreas del conocimiento, incluso en donde tiene menos participación femenina. Llama la atención esto último, ya que las áreas con menor matrícula femenina presentan aumento en el número de titulaciones femenina

De acuerdo a la información estadística brindada por las instituciones de educación superior, durante el año 2014 se entregaron 23,194 títulos o grados terminales, de los cuales, el $56.39 \%$ corresponden a mujeres $(13,079)$ y solo el $43.61 \%$ a hombres $(10,115)$.

Haciendo una comparación para el año 2010 se tuvo una titulación femenina en nivel universitario de $61.14 \%$, aunque es una diferencia de 22 puntos porcentuales de la brecha de género, la cual se hace más corta debido a que en el año 2014 la titulación femenina en todas las áreas fue menor; con un $58.61 \%$ y 17 puntos porcentuales.

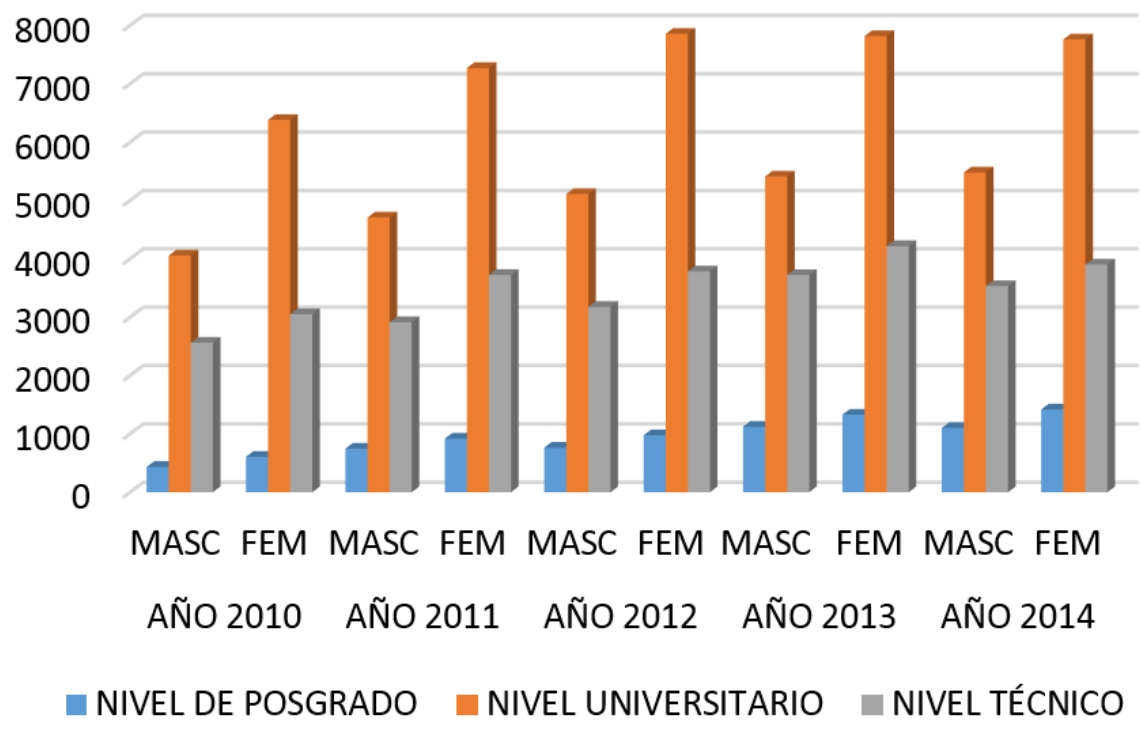

Gráfico n. ${ }^{\circ}$ 5. Titulación según grado académico y género. Fuente: Elaboración propia con datos estadísticos del Ministerio de Educación 2010-2014.

Existen importantes diferencias en cuanto al nivel de estudios que alcanzan ambos sexos. En el Gráfico n. 5 se advierte que las mujeres han llegado y sobrepasado a la paridad en la obtención de títulos universitarios.
Para Altbach (2016) la idea de que un grado académico representa un bien privado que beneficia a los individuos, más que un bien público para beneficio de la sociedad, es ampliamente aceptada. En países asiáticos como 
Japón, Corea, Filipinas e Indonesia, la educación superior privada domina a la pública en términos de números.

En El Salvador se da un caso similar con el surgimiento de las instituciones de educación superior privada que desde el año 1981. A raíz del conflicto armado prevalente en el país. En la actualidad, El Salvador cuenta con ocho instituciones de educación superior del sector público (una universidad, cuatro institutos especializados y tres institutos tecnológicos) y 40 IES del sector privado (24 universidades, 9 institutos especializados y 7 institutos tecnológicos).
Las IES públicas recibieron en concepto de subsidio del Estado 2010 según Ministerio de Educación (2014) un monto presupuestario de $\$ 73,122,967.12$ y para 2014 tuvo un aumento de $23.93 \%$ con un monto de $\$ 90,624,394.88$, pasando de una inversión anual del estado por estudiante de $\$ 1,457.47$ al $2014 \$ 1,666.72$, pero con todo y el subsidio que da el Estado a las IES no es suficiente por el número de estudiantes que quieren hacer uso a la educación superior, lo que conlleva a que el 69\% de titulaciones para el 2014 estuvieron dadas por las IES del sector privado como lo muestra en el Gráfico n. ${ }^{\circ} 6$.

TITULACIÓN POR GÉNERO Y SECTOR PÚBLICO Y PRIVADO, PERÍODO 2010 - 2014

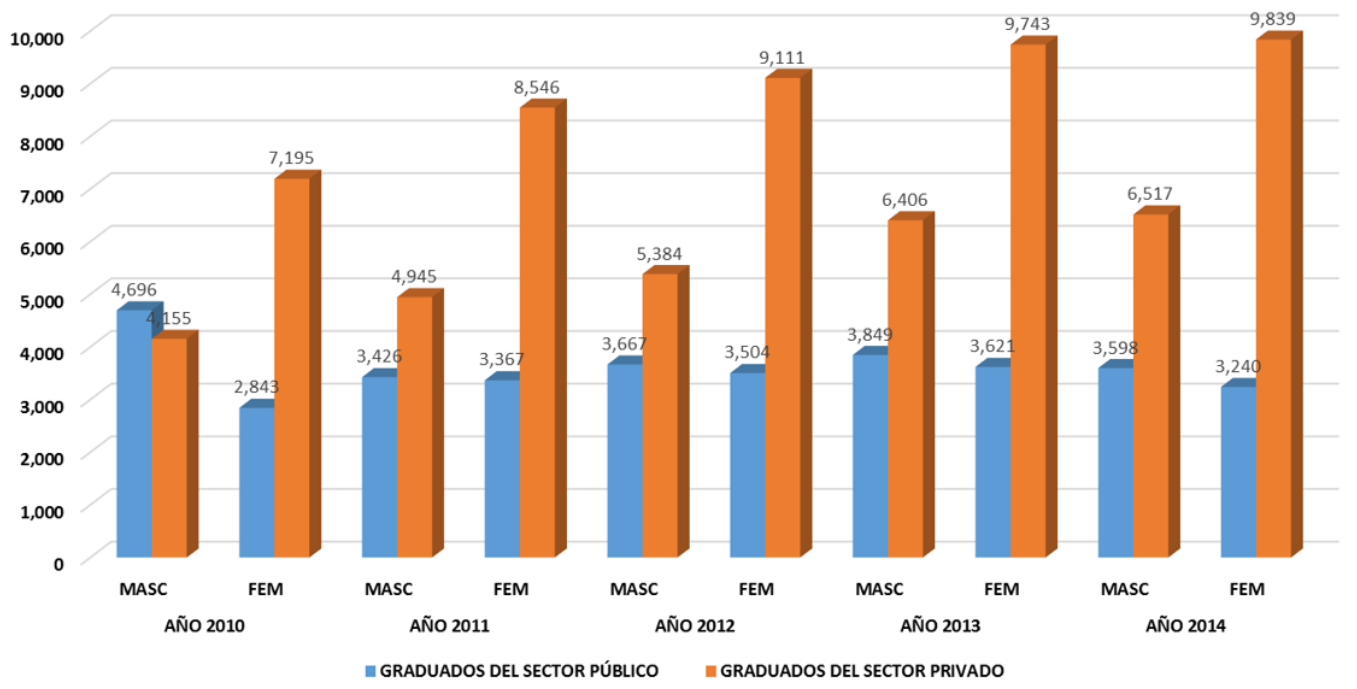

Gráfico n. ${ }^{\circ}$ 6. Titulación por género y sector público y privado. Fuente: Elaboración propia con datos estadísticos del Ministerio de Educación 2010-2014

\section{Área de Posgrado: Matrícula}

En el quinquenio en estudio se analiza la matrícula de posgrado (Gráfico n. ${ }^{\circ}$ 7) observando una tendencia muy marcada a favor del género femenino que culmina en la equidad por género de la matrícula, que va desde el año 2010 al 2014. 


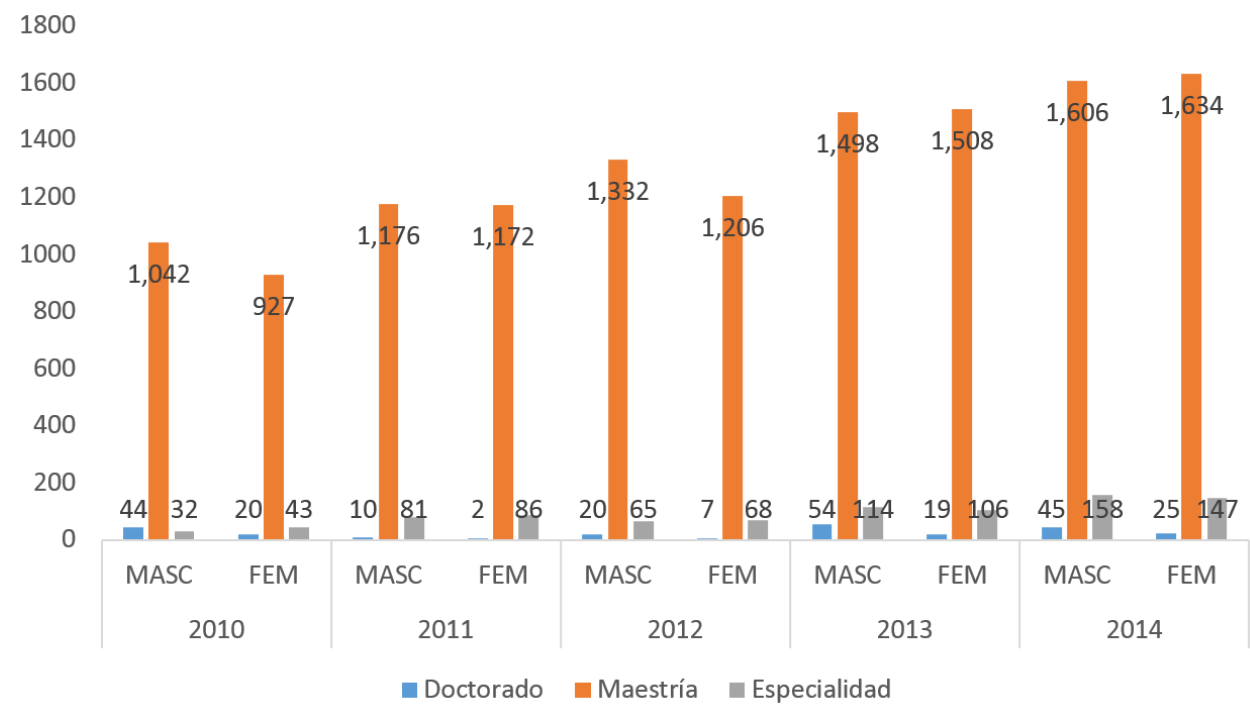

Gráfico n. ${ }^{\circ} 7$. Matrícula de posgrado según titulación y género. Fuente: Elaboración propia con datos estadísticos del Ministerio de Educación 2010-2014

\section{Titulación Posgrado}

En relación a la Titulación general del posgrado (Gráfico n. ${ }^{\circ}$ ), similar a la matrícula, hay una tendencia a la equidad de la titulación, favoreciendo al género femenino. Aunque debemos aclarar que si observamos solo la titulación del doctorado, la tendencia favorece al género masculino, caso contrario a lo que sucede con la titulación de los estudios de maestría y especialización.

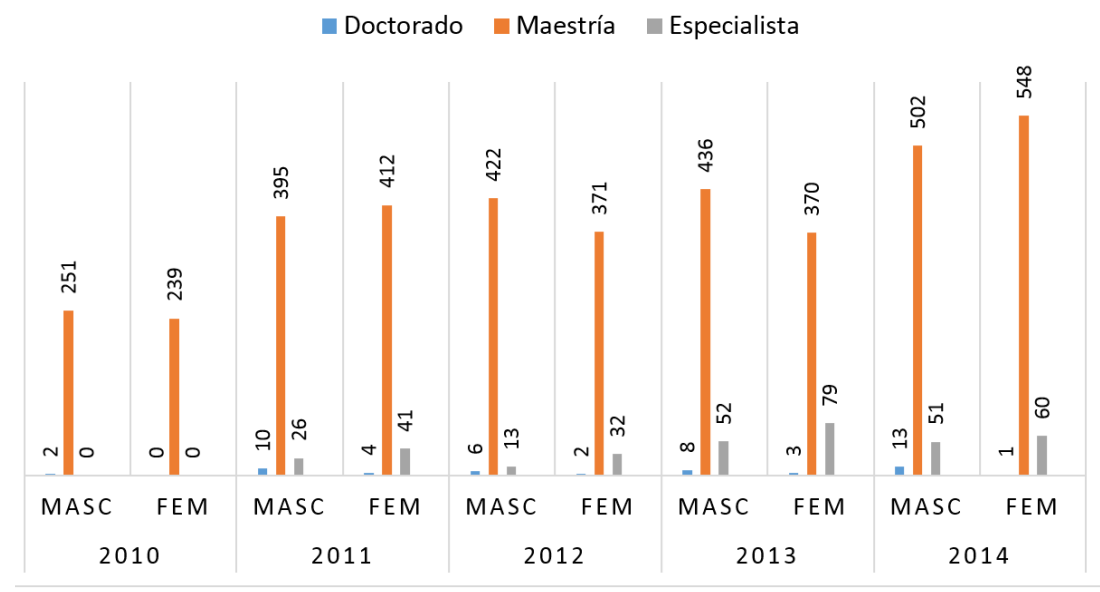

Gráfico n. ${ }^{\circ}$. Titulación a nivel de posgrado según género. Fuente: Elaboración propia con datos estadísticos del Ministerio de Educación 2010-2014. 


\section{Planta docente por género y titulación}

Desde el año 1998 en la Conferencia Mundial de Educación Superior señalan y hacen hincapié en la importancia de aumentar la presencia de la mujer tanto en el personal como en el alumnado supone un gran desafío para las instituciones. En lo relativo al alumnado, esto se ha cumplido en El Salvador. El desafío es aún mayor para aquellos que tratan de lograr una mayor participación de la mujer en la planta docente y la titulación de posgrado, en los puestos de alto nivel de la docencia, la investigación o la gestión institucional. Como se puede observar en la Tabla n. ${ }^{\circ}$, la marcada tendencia a la masculinización de la planta docente a nivel superior con posgrado a nivel de maestrías, especialidad y doctorado.

Tabla n. ${ }^{\circ} 3$

Planta docente por género y titulación de posgrado

\begin{tabular}{|c|c|c|c|c|c|c|c|c|c|c|c|c|c|c|c|}
\hline \multirow{2}{*}{ GRADO ACADÉMICO } & \multicolumn{3}{|c|}{ AÑO 2010} & \multicolumn{3}{|c|}{ AÑO 2011} & \multicolumn{3}{|c|}{ AÑO 2012} & \multicolumn{3}{|c|}{ AÑO 2013} & \multicolumn{3}{|c|}{ AÑO 2014} \\
\hline & MASC. & FEM. & TOTAL & MASC. & FEM. & TOTAL & MASC. & FEM. & TOTAL & MASC. & FEM. & TOTAL & MASC. & FEM. & TOTAL \\
\hline DOCTORADO PG. & 158 & 33 & 191 & 156 & 31 & 187 & 140 & 33 & 173 & 144 & 37 & 181 & 163 & 37 & 200 \\
\hline ESPECIALIDAD & 163 & 100 & 263 & 100 & 51 & 151 & 99 & 54 & 153 & 127 & 62 & 189 & 140 & 70 & 210 \\
\hline MAESTRÍA & 1,270 & 658 & 1,928 & 1,407 & 765 & 2,172 & 1,419 & 829 & 2,248 & 1,422 & 771 & 2,193 & 1,493 & 840 & 2,333 \\
\hline TOTAL & 1,591 & 791 & 2,382 & 1,663 & 847 & 2,510 & 1,658 & 916 & 2,574 & 1,693 & 870 & 2,563 & 1796 & 947 & 2743 \\
\hline PORCENTAJE & 66.79 & 33.20 & 100 & 66.25 & 33.74 & 100 & 64.41 & 35.58 & 100.0 & 66.05 & 33.94 & 100 & 65.47 & 34.52 & 100 \\
\hline
\end{tabular}

Fuente: Elaboración propia con datos estadísticos del Ministerio de Educación 2010-2014.

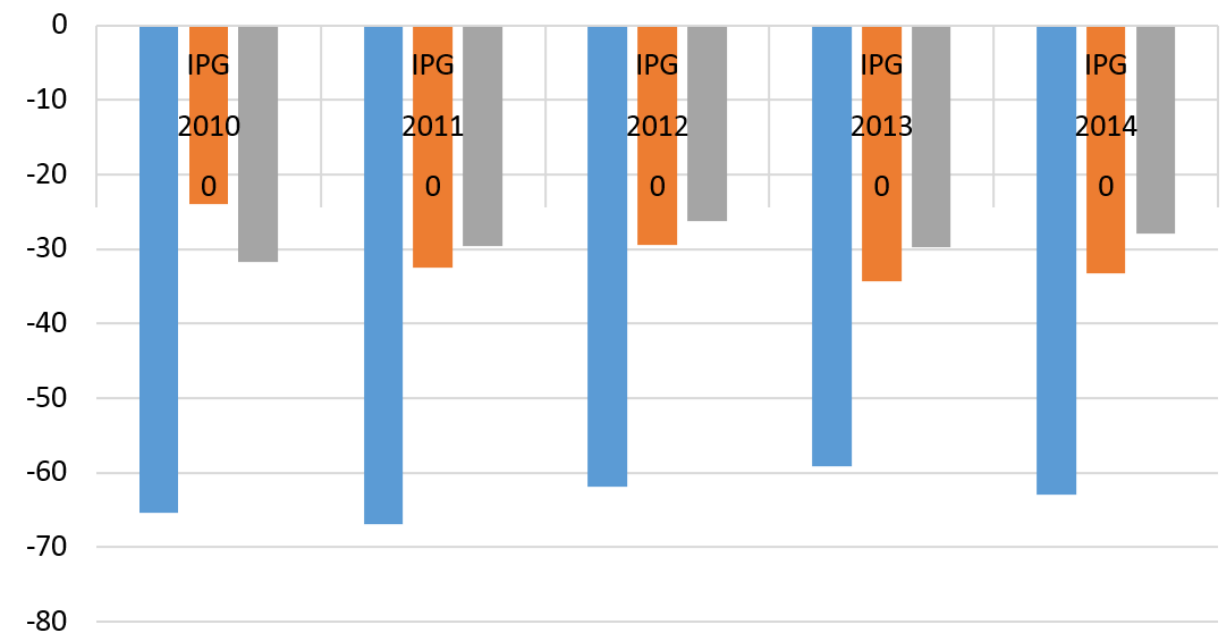

\section{- DOCTORADO PG. ESPECIALIDAD MAESTRÍA}

Gráfico n. ${ }^{\circ}$ 9. Planta docente según titulación (posgrado) y género. Fuente: Elaboración propia con datos estadísticos del Ministerio de Educación 2010-2014. 
En el Gráfico n. ${ }^{\circ}$, llama la atención que a lo largo de este análisis, tanto en matrícula, titulación y en el Índice de Paridad de Género se denota una tendencia que favorece al género femenino; por lo tanto se esperaría que la feminización de la planta docente de primaria reflejara una tendencia similar, pero contrario a los esperado, el IPG es bajo, siendo en el personal docentes los estudios de posgrado con marcada tendencia al género masculino.

\section{Conclusiones}

La feminización de la matrícula y titulación de pregrado no significa que en la región se presente una feminización de los programas; según el análisis, las mujeres ingresan a carreras conocidas tradicionalmente como femeninas: educación, áreas de la salud y ciencias sociales. Se muestra una pequeña variación en carreras típicamente masculinas como: ingenierías, las científicas y tecnológicas. Esta distinción lleva a pensar en una continuidad en cuanto a los roles socialmente ubicados como del ámbito doméstico.

Aun cuando a la matrícula y titulación de posgrado se analizó que en El Salvador hablando de una perspectiva de género muestra una equidad aparente en los números absolutos, pero si observamos a nivel de doctorados esta tendencia favorece al género masculino

En cuanto a la docencia, hay una diferencia en la vinculación a la educación superior marcada por el género, el mayor porcentaje está a favor de los hombres en las instituciones de educación superior, al pasar los años el porcentaje se mantiene casi idéntico. En donde la participación de la planta docente universitaria en consonancia con la tendencia sigue siendo masculina, más aun cuando se analiza según grado la titulación de Posgrado.

\section{Referencias bibliográficas}

Altbach, P. (2016). Global Perspectives on Higher Education. Baltimore: Johns Hopkins University Press.

Busto, O. (2004). Reordenamientos genéricos de la matrícula en la educación superior. El caso de México. Otras miradas, 4, pp. 3049. Recuperado de: http://www.redalyc.org/ articulo.oa?id $=18340104$

Ministerio de Educación República de El Salvador (2014). Educación Superior en cifras, El Salvador 2002-2012. San Salvador: Imprenta Nacional.

Ministerio de Educación República de El Salvador (2015). Resultados de la Información Estadística de Instituciones de Educación Superior 2014. San Salvador: Imprenta Nacional.

Ministerio de Educación República de El Salvador (2014). Resultados de la Información Estadística de Instituciones de Educación Superior 2013. San Salvador: Imprenta Nacional.

Ministerio de Educación República de El Salvador (2013). Resultados de la Información Estadística de Instituciones de Educación Superior 2012. San Salvador: Imprenta Nacional.

Organización de las Naciones Unidas para la Educación, la Ciencia y la Cultura (UNESCO) (2009). Indicadores de la Educación. Especificaciones técnicas. Montreal: Autor. 
Organización de las Naciones Unidas para la Educación, la Ciencia y la Cultura (UNESCO) (2012). Atlas Mundial de la Igualdad de género en la Educación. París: Autor. Recuperado de: http://unesdoc.unesco. org/images/0021/002155/215522E.pdf

Papadópulos, J.y Radakovich R. (2006). Educación Superior y Género en América Latina y el Caribe, en: Informe sobre la Educación Superior en América Latina y el Caribe 2000-2005. Caracas: IESALC. Recuperado de: www.iesalc.unesco.org.ve/ index.php?option=com_fabrik...
Sierra, R. y Rodríguez, G. (2005). Feminización de la matricula de educación superior en América Latina y el Caribe. México D.F.: IESALC / UNESCO. Unión de Universidades de América Latina, UDUAL, pp. 468. 